\title{
ENTRPERENEURIAL BUSINESS LINKAGES VIA STRATEGIC INFORMATION SYSTEMS
}

\author{
Brian Mackie, Northern Illinois University, bmackie@niu.edu \\ Norbert Ziemer, Northern Illinois University,nziemer@niu.edu
}

\begin{abstract}
Entrepreneurship and economic development are desirable for encouraging new business development, existing business growth and positive impacts on regions. However, the process of achieving these outcomes is challenging and not always straightforward. A method of systematizing information assets holds the potential to help one business benefit from another business. The authors have developed a plan to capture and manage pertinent information to identify new market and supply chain opportunities by using techniques that go beyond traditional industry classifications and standard economic development information.
\end{abstract}

Keywords: Strategic Information, Entrepreneurial Matchmaking, Supply Chain Linkages

\section{INTRODUCTION}

Economic development and entrepreneurial capacity are key factors for encouraging new business development, existing business growth and positive impacts on regions. The process of achieving these outcomes, though, is challenging and not necessarily straightforward. Multiple regions and the economic development organizations serving them compete to draw new firms to locate in their respective geographical districts. Today, these organizations have access to an array of information sources. This research develops a framework implemented using a database that can enable rapid identification of opportunities that allow two companies to benefit from each other's assets. This is designed to go beyond traditional mergers, acquisitions, transactions and interactions through industry classifications and standard economic development information.

\section{Entrepreneurship and Economic Development}

Entrepreneurs convert and rearrange resources in order to increase the overall value. This is often done through new products although it can take the form of services and even new business models. It is the business of the entrepreneur to determine which projects will be profitable [3]. Central to entrepreneurship is the innovation process, in which something new is created. A number of factors such as increased economic globalization have further propelled interest in entrepreneurship and entrepreneurial activity.

Entrepreneurship literature has covered elements relevant to this research. In addition it is advantageous to have knowledge of the more technical aspects as well as the business aspects, value chain elements, and the market landscape. Social networks and information management are tools which may support technology entrepreneurs and enhance economic development efforts.

\section{Business Incubators to Stimulate Technology Based Entrepreneur Development}

Business incubators are one approach used for economic development and technology entrepreneurship. Incubators can have a positive overall impact on the success of new business development efforts. One of the underlying factors believed to impact the effectiveness of business incubators is the increase in interpersonal and interfirm connections made available to entrepreneurs within the incubator. These interactions can lead to mutually beneficial outcomes for the two or more parties involved. Additionally, access to key information is essential in order to have an effective strategic planning process for new venture or new product development efforts. Incubators also tend to 


\section{Issues in Information Systems}

Volume 13, Issue 2, pp. 361-368, 2012

increase the information exchange for tenant firms and entrepreneurs, allowing involved parties to pool assets and develop mutual synergies on specific projects or ventures.

\section{RESEARCH PROPOSITION}

This research illustrates the impetus for and the development of a system to match businesses There is potential to link information, contacts, 'aha moments' and innovative ideas in a manner similar to bricks and mortar incubators; this is desirable since it may help spur new business opportunities and may do so with less capital outlay than traditional incubators. It can be used to find obvious matches, like many commercially available business information systems do; however, it can additionally be used to help identify potential matches which are not obvious. By adding this capability, the system will have an increased likelihood of generating new, non-obvious matches as may occur in a business incubator. As it stands, the system is in its second iteration of the prototype.

This system combines process and technology for enhanced information management with the intent of positively impacting entrepreneurial opportunity recognition. A unique process for interviewing companies has been developed in which a trained interviewer will draw out a high degree of useful information that will be used in the matching process. The interview captures a wide range of information about the firm, its present business operations, developments and challenges, as well as about areas of emerging interest for the firm's future.

This information is then warehoused within a database. The information technology capabilities of the database system will leverage the rich information gleaned from the interview process to extend the system far beyond the capabilities of a conventional contact database. The system will use this information to help increase opportunity recognition. The system is able to automatically search and identify unique matches which would not found by typical means such as SIC or NAICS codes used in economic development classification. The queries for possible matches will be run at pre-programmed times, for example at night. Then, when the executive/manager returns to work in the morning, he or she will see a display of new potential strategic matches. The manager would then take action and use his or her judgment to reach out the firms, explaining that they may benefit from a linkage to the other firm, and then ask permission to introduce the two firms and speak to the opportunity. Also, as the database grows, the increased members and updated information will lead to an increased number of hits or "matches" for even more opportunities for business collaboration.

The system uses process and technology to glean rich information about entrepreneurial firms and their respective resources [8]. The system's automated match identification replaces the tradition labor-intensive and resourceintensive approach which would include manually identifying potential business-to-business matches for developing organizational and supply chain synergies. This allows the economic development organization to expand entrepreneurial efforts that will result in new economic activity.

This research is built upon foundations from the literature related to entrepreneurial opportunity recognition and knowledge [7]. It extends some of the theory from business incubators and economic development efforts and incorporates an innovative information systems approach to increase new economic activity. Fundamentally, once this system is fully implemented with data from many companies, it will generate more opportunities for new use, innovation and/or entrepreneurial deployment. These opportunities may relate to new startup businesses as well as uncover supply chain and operational advantages for existing firms.

\section{METHODOLOGY}

The approach incorporates both process and information technology innovation. The procedure includes gathering information from companies, evaluating that information, finding possible matches and then introducing the companies that have a higher match quotient.

A chronological outline of events is as follows:

- Locate companies 


\section{Issues in Information Systems}

Volume 13, Issue 2, pp. 361-368, 2012

- $\quad$ Ask them for the opportunity to interview

- Economic development organization contacts companies to ascertain their willingness to have an interviewer conduct a site visit

- The economic development professional coordinates the date of the site visit

- Economic development professional requests video/audio augment augmentation

- Explain why this procedure is important and how outcomes can benefit the company

- Provides more in-depth picture of assets and liabilities

- Specify and identify markets

- Highlight "useful waste"

- A pre-assessment is conducted

○ Talk with the company about their needs and/or wants

- This helps make the site visit more effective

- It is recorded to allow better translation when the total interaction is debriefed and later put into the companies match criteria profile

At the site visit the interviewer starts by talking with the company and expanding on the information given during the pre-site interview. Many of the company's hopes and wishes are discussed. Next the interviewer takes a tour of the facility notating human resources and limitations, material surpluses and other shortages. Waste products are identified and cost of disposal is documented. Special attention is given to the responses here as the information provided at this stage can translate into the identification of new opportunities for value creation.

After the site visit the economic development professional begins to compile all of the information known about the firm to develop a composite. This includes the pre-site discourse, the site interview information, and the notes from the tour. This information is then transcribed into a list of surplus resources and resources that are desired or would be beneficial and any resources or waste that could be used by others. Also recorded are any potential problems or strategic vulnerabilities which may be of concern in the future. This information will then be entered into the company's match data profile.

The process explained earlier begins with a "Voice of the Customer" session [2]. This will help the interviewer to glean rich information that will be added to the database.

Beyond the stock questions included in the "Voice of the Customer" interview, additional questions that will be asked include:

- "What specific resources would your organization want more access to?"

- "What unused materials/products (e.g. waste, production scrap) does your operation have?"

- "What capabilities and/or capital does your firm have that are not being fully utilized?"

- "What operational 'leftovers' or 'co-products' does your firm have?"

The procedure will enable the interviewer to glean very useful information vital to populating and growing the database. The site visit will ideally be made with the Chief Executive Officer, Chief Operations Officer, or the like. The process and information management approach will help enhance the coordination of manufacturing and production relative to markets and supply chain considerations [1].

Overall, these and many other questions help draw out useful information during the interview to help identity gaps and opportunities for value chain development and management. During the site visit the interviewer will take note of opportunities and needs that may benefit the business and try to discover possible waste products that may have potential to benefit another organization. The resources may include human capital, surplus product, production scrap or co-products, equipment and physical assets. All of these may be assets that are underutilized or no longer needed by the firm. 


\section{Issues in Information Systems}

Volume 13, Issue 2, pp. 361-368, 2012

The information gathering process will ideally include at least one and likely more site visits to the organization. In rare cases, a virtual tour done by a specially trained interviewer may provide initial information about the organizations needs and waste. Interviewers will receive training to maximize the potential for rich information gathering, as well as to provide a consistent framework for the interview process. The process of walking through the business environment can be highly informative to an observant visitor. The results will reflect the interviewer's ability to draw out information from the organization.

\section{RESULTS}

In its present state the system is functional and has some company data. The database has been configured to incorporate rich information. The categories can be easily expanded as the scope and breadth of the firms and the types of classification for the information retrieved grow. Figure 1 displays the main system menu. This menu includes a link to the administrative menu shown in Figure four and the master table link which takes the user to the screen displayed in Figure 2.

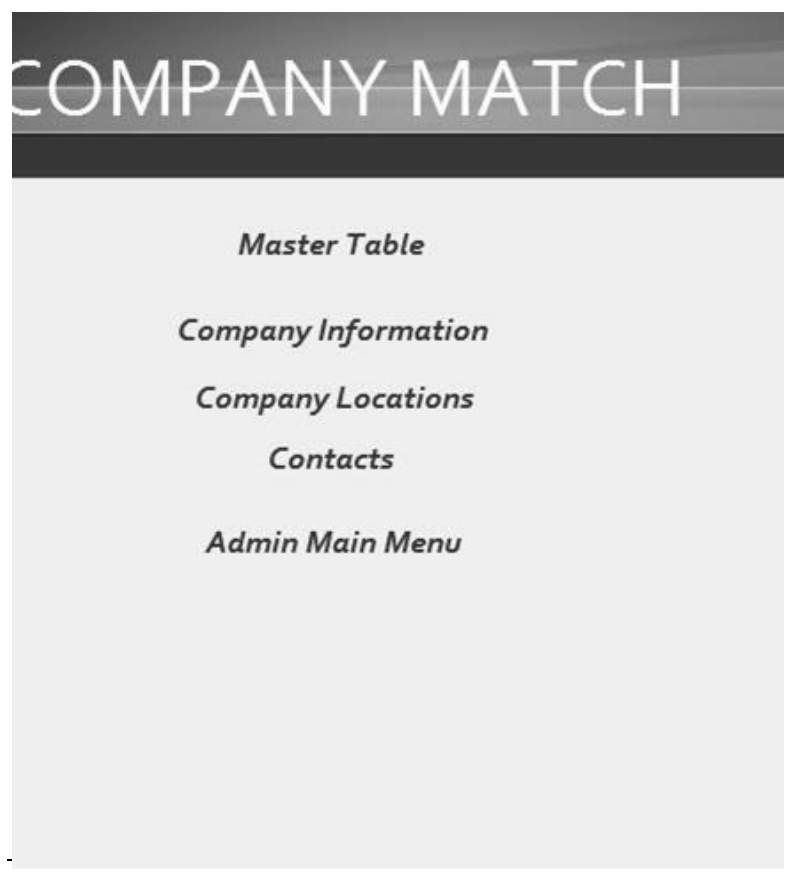

Figure 1. Initial Screen

The screen shown in Figure 2 enables access to organizational information and the locations for that organization. It also allows the entry of a new organizations and/or new locations. Once the organization and location is selected the screen displayed in Figure 3 is displayed. This enables the system operator to begin to populate the system with company information gleaned from the pre-assessment and the site visit. 


\section{Issues in Information Systems}

Volume 13, Issue 2, pp. 361-368, 2012

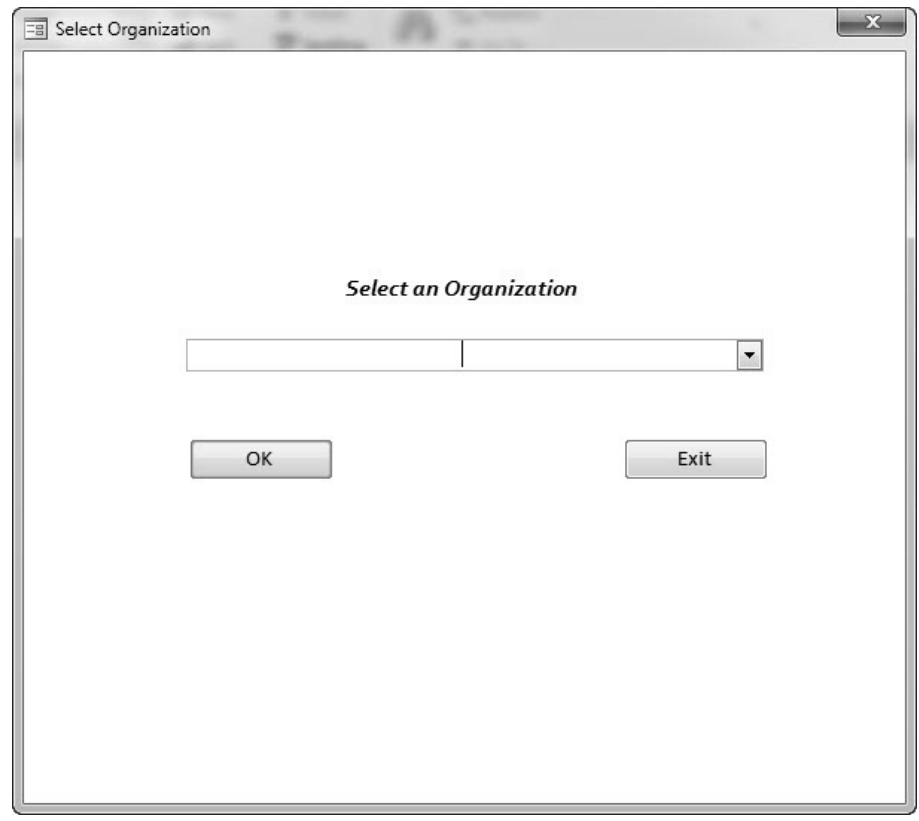

Figure 2. Company Match Information

To benefit the reader, a couple of representative examples are included. One is a lead originated by a food processing organization. This is a prominent firm with a well-established product line. As with many organizations today, this firm is seeking to be better stewards of the resources being utilized in its operations. One metric toward this end is the reduction of non-value added outputs leaving the production facility. One main co-product that is currently leaving the facility in a non-value added state is part of the food cuts, which are vegetable based. This biomass product may have many potential realms for new use applications: food, feed, fiber, fuel/energy, and fertilizer. One of the matches for this product is a prominent agricultural production enterprise in the region. This producer has been evaluating the reduction of synthetic fertilizers on its farms through increased application of more biorenewable fertilizers and soil amendments. Thus, the food co-product from the first firm may be a beneficial component when added into the biobased compost soil treatment of the second firm; this type of business relationship becomes a mutually beneficial exchange.

A second example focuses more on the human capital part of the operation. A firm has a skilled employee who has been with the firm for several years and has intellectual capital that this firm does not want to lose. Current economic conditions and the resultant reduction in business orders have increased pressure on this firm to reduce operational costs. The firm does not want to lose this employee and could employ her at a half-time capacity. A match is found with another local firm that has just received a significant production contract and this firm wants to expand at least temporarily to match this spike in demand. However, the second firm is aware of the highly variable nature of its business cycles, so it prefers to hire some of its workforce as temporary workers. The information mentioned above would be stored as entries in the match table displayed in Figure 3. These firms are geographically close together, so travel is not an issue. This match could prove beneficial to both firms and to the worker. 


\section{Issues in Information Systems}

Volume 13, Issue 2, pp. 361-368, 2012

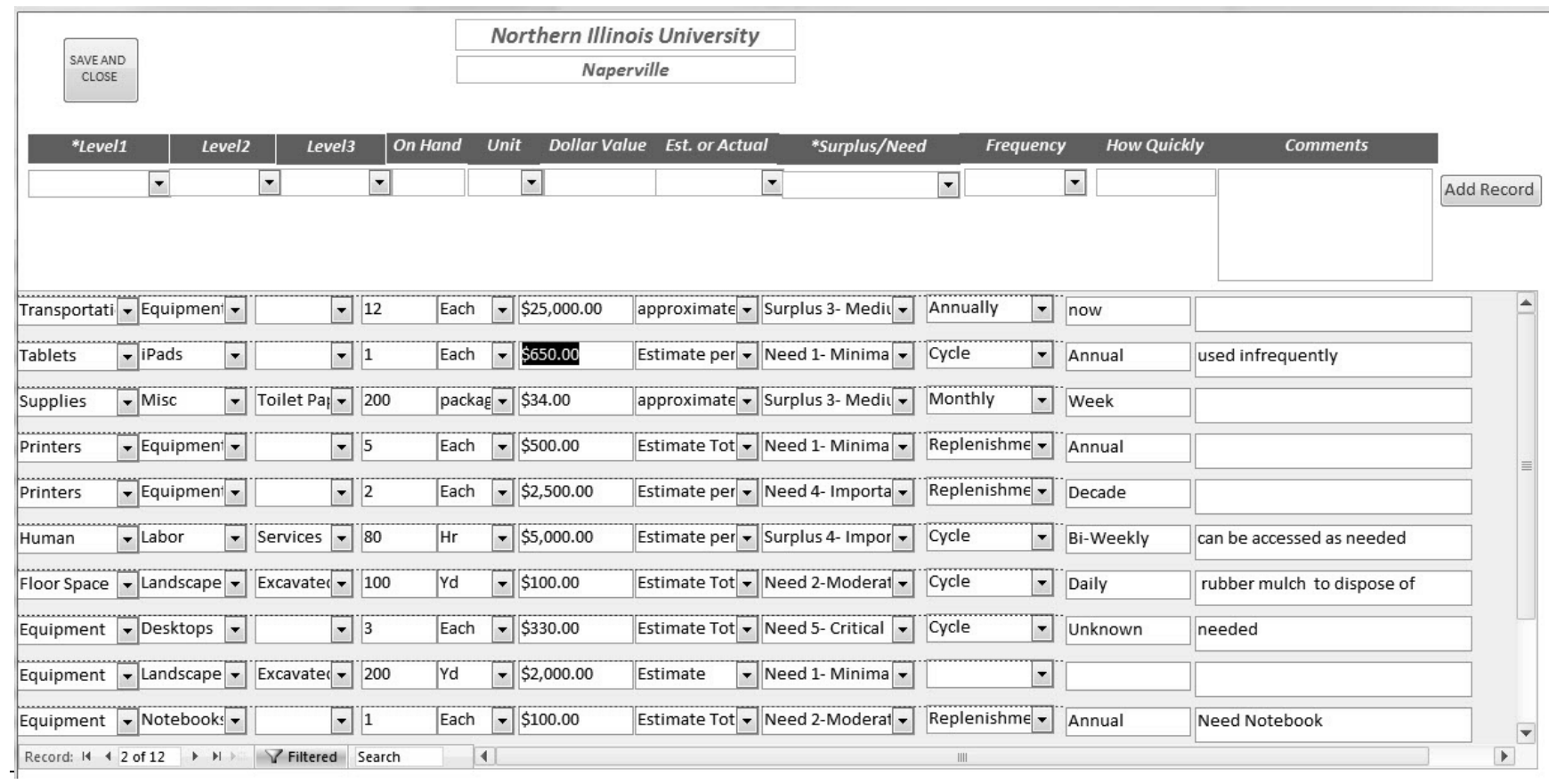

Figure 3. Company Match Expanded Information

Figure 1 and Figure 4 give you links to many of the capabilities of the system. Here, the operator is able to add and update organizations as well as manage information for multiple locations and business units for a given corporation. The operator has the option of using the automated functionality of the system. Additionally, the operator is empowered to manually run the match for all organizations or a select group, in order to find their level of commonality.

Figure 3 is a screen capture of the database illustrating how the information is managed. This includes how the information is populated into the database and how the information management is structured. There are three levels of categories. There are also numerical inputs for number of units and unit values. A ranking system captures both the direction (need or surplus) and the ranking of the level of importance of the need or surplus. Additional criteria can also be added to the system. 


\section{Issues in Information Systems}

Volume 13, Issue 2, pp. 361-368, 2012

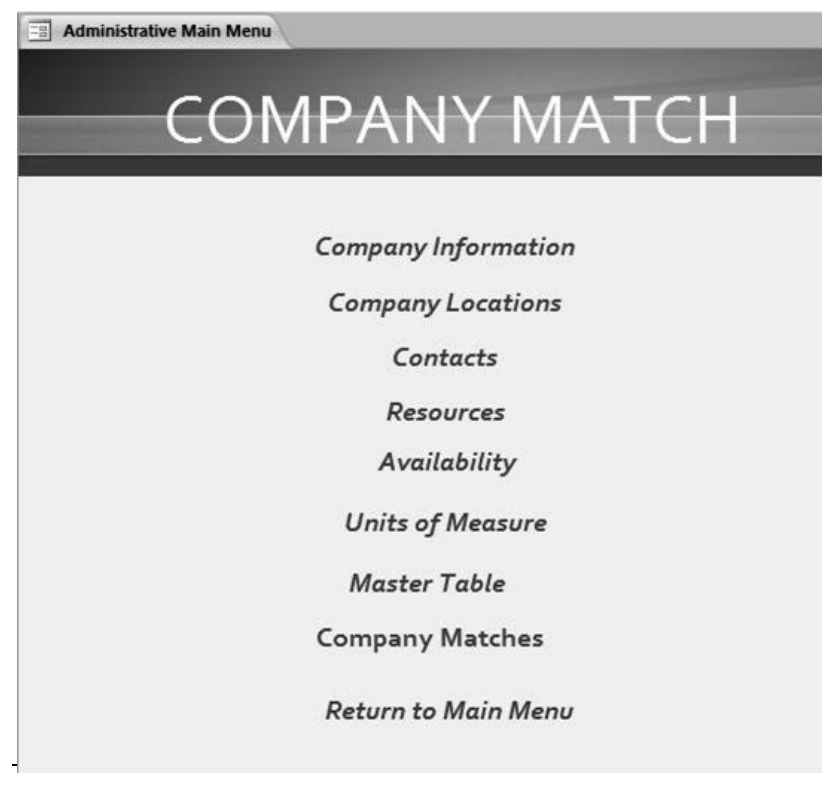

Figure 4. Company Matches

The operator will have the option of using the automated functionality of the system, as shown in Figure 4.

Additionally, the operator is empowered to manually run the match for all organizations or a select group, in order to find their level of commonality.

The resulting information tells why the system is matching two organizations. Preset criteria serve as a decision tool, based on weighted inputs on the matching process. For example, there may be a predetermined minimum requiring four key criteria to be met and an overall $86 \%$ threshold value. If two organizations meet the criteria and threshold they would be given the opportunity to be introduced.

\section{CONCLUSIONS AND FUTURE RESEARCH}

Conceptually, this is an innovative approach to increasing the rate of business connections with the purpose of helping to allocate or reallocate resources and assets to be deployed more effectively, through the use of Information Technology to enhance the firm's core competencies [6]. The authors began a prototype implementation. Currently, the system is being expanded and improved before deploying it on a larger scale. Feedback is being gathered and observations being made to iteratively improve the system [5]. Lastly, the system has the capability of increasing the rate of opportunity recognition, which is a factor closely aligned with entrepreneurship [4].This will allow economic development organizations, working to spur entrepreneurship and new business opportunities, to effectively do "more with less." 


\section{Issues in Information Systems}

Volume 13, Issue 2, pp. 361-368, 2012

\section{REFERENCES}

1. Bharadwaj, S, Bharadwaj, A. \& Bendoly, E. (2007). The Performance Effects of Complementarities between Information Systems, Marketing, Manufacturing, and Supply Chain Processes. Information Systems Research, $18,4$.

2. Griffin, A. \& Hauser, J.R. (1993). The Voice of the Customer. Marketing Science, 12, 1.

3. Gwartney, James D., Stroup, R.L. \& Sobel, R.S. (2000). Economics: Private and Public Choice. Ninth edition, The Dryden Press.

4. Hills, G.E., Shrader, R.C., \& Lumpkin, G.T. (1999). Opportunity recognition as a creative process. Frontiers of entrepreneurship research, 19, 1926. Babson College.

5. Mackie, B.G, Ziemer, N.L., Russo, N.L., \& Mackie, (2004). W.E. Developing a Homegrown Course Management System - Community/course, Action/interaction Management System (CAMS). Journal of Cases on Information Technology. 6, 1 .

6. Ravichandran, T., Lertwongsatien, C., \& Lertwongsatien, C. (2005). Effect of Information Systems Resources and Capabilities on Firm Performance: A Resource-Based Perspective, 21, 4.

7. Shane, S. (2000). Prior knowledge and the discovery of entrepreneurial opportunities. Organization Science, 11,4 .

8. Wade, M. \& Hulland, J. (2004). The Resource-Based View and Information Systems Research: Review, Extension, and Suggestions for Future Research, 28, 1. 